\title{
OBSERVAÇÃO MICROSCÓPICA DE INCLUSÕES MINERAIS NO XILEMA DE ESPÉCIES TROPICAIS DA AMAZÔNIA.
}

\author{
Francisco José de VASCONCELLOS ${ }^{1}$, Jorge Alves de FREITAS ${ }^{\mathfrak{1}}$, Ademir \\ Castro e SILVA ${ }^{2}$
}

\begin{abstract}
RESUMO - Foi investigada a distribuição de inclusões minerais (cristais e grãos de snica) no xilema secundário de 53 famílias, representando 250 espécies de madeiras amazônicas. Grãos de sílica ocorrem em cerca de $36 \%$ das espécies estudadas, frequentemente nas células dos raios. Cristais nas células dos raios são geralmente solitários, enquanto que nas fibras e células do parênquima axial são algumas vezes em cadeias cristalíferas. Os cristais são frequentemente do tipo prismático e somente em uma espécie (Scleronema micranthum) foi encontrado cristal do tipo drusa.
\end{abstract}

Palavras-chaves: Madeiras da Amazônia, inclusões minerais, sílica, cristais.

Microscopical Observation of Mineral Inclusions in Xylem of Tropical Wood.

ABSTRACT - The distribuition of mineral inclusions (crystals and silica grains) in the secondary xylem of 250 species representing 53 families of amazonian woods was investigated. Silica grains occur in about $36 \%$ of the species studied. They are most frequently found in the ray cells almost always are solitary while in the fibers and parenchyma cells are sometimes in crystalliferous chains. The crystals are frequently of prismatic type and only in one species (Scleronema micranthum) was found crystal of druse's type.

Key-words: Amazonian woods, mineral inclusions, silica, crystals.

\section{INTRODUÇÃO}

A presença de inclusões minerais (cristais e grãos de sílica) em madeiras tem sido motivo de estudos em várias partes do mundo (RECORD, 1927; BAMBER \& LANYON, 1960; SHARMA \& RAO, 1970; SCURFIELD et al, 1974; WELLE, 1976; LAROCHE, 1977; KOEPPEN, 1980).

Alguns estudos correlacionam a presença dessas incrustações à avaliação taxonômica, enquanto outros avaliam a possível correlação entre a sua presença e as dificuldades encontradas no beneficiamento da madeira. Sabe-se que a presença de sílica dificulta o desdobro da tora, devido ao seu efeito abrasivo sobre os dentes das serras, fazendo com que as mesmas sejam levadas ao setor de laminação da indústria mais vezes do que o usual para a devida afiação. Utilizando-se aço especial e executandose a serragem das toras que previamente condicionadas na água, pode-se minimizar tal problema. Entretanto, antes de iniciarmos tal procedimento é imprescindivel conhecer quais as madeiras que apresentam tais incrustações. Considerando que tais informações para madeiras tropicais são raras e dispersas na literatura, iniciou-se estudos sobre $o$ assunto para as famílias pertencentes ao grupo das Leguminosas (VASCONCELLOS et al., 1993; FREITAS et al., 1992).

1 Instituto Nacional de Pesquisas da Amazônia - INPA Av. André Araújo, 1756 - Aleixo, 69083-000 - Manaus/Am.

2 Centro de Ensino e Pesquisas Florestais - CEPEF/UTAM Av. Darcy Vargas, 1200 - 69055020 - Manaus/Am. 
Sílica também é utilizada como característica para se diferenciar gêneros e espécies. RICHTER (1982), por exemplo, separa os gêneros Couropita de Couratari (Lecythidaceae), cujas madeiras são similares em aparência e indistinguíveis macroscopicamente, pela ocorrência de grãos de sílica no xilema de Couratari. Do mesmo modo a presença de cristais pode ser uma característica para diferenciação de espécies dentro de um mesmo gênero (VASCONCELLOS et al., 1993; FREITAS et al., 1992). A presença de séries cristalíferas tem sido utilizada como indicador de idade em espécies africanas de Acacia (GOURLAY \& KANOWSKI, 1991).

Assim, o presente trabalho pretende examinar o xilema de espécies tropicais pertencentes a 52 famílias, visando detectar a presença de sílica e cristais e sua localização nos diversos elementos xilemáticos.

\section{MATERIAL E MÉTODOS}

O material examinado nesta pesquisa encontra-se registrado e devidamente acondicionado no laminário do Laboratório de Anatomia e Identificação de Madeiras da Coordenação de Pesquisas em Produtos Florestais do Instituto Nacional de Pesquisas da Amazônia - CPPF/INPA. Foram observadas as lâminas das seguintes espécies de madeiras: ANACARDIACEAE (Astronium lecointei Ducke); ANNONACEAE (Annona sericea Dun; Bocageopsis multiflora R.E. Fries; Duguetia quitarensis Benth; Duguetia ulei (Diels)
R.E. Fries; Ephedranthus amazonicus R.E. Fries; Guatteria scytophylla Diels; Pseudoxandra polypheba R.E. Fries; Rollinia insignis R.E. Fries; Unonopsis floribunda Diels; Unonopsis guatterioides R.E. Fries; Xylopia aromatica (Lam.) Mart.; Xylopia benthamii R.E. Fries; Xylopia cf. calophylla R.E. Fries); ANTONIACEAE (Antonia ovata Phol.); APOCYNACEAE (Ambelania quadrangulares Muell. Arg.; Ambelania tenuiflora Muell. Arg; Aspidosperma album R. Benth; Aspidosperma obscurinervium Azambuja; Aspidosperma oblongum A. DC; Couma guianensis Aubl; Couma utilis Muell. Arg.; Hymatanthus attenuata Woodson; Hymatanthus sucuuba Woodson; Lacmellea cf. lactescens Kullm; Macoubea guianensis Aubl; Macoubea sprucei Mgf.; Maloetia duckei Mgf.; Neocouma ternstroeniaceae (Muell. Arg.) Pierre; Rauwolfia duckei M.G.F.; Rauwolfia pentaphylla Ducke; Rauwofia sellowii Muell. Arg.; Zschokkea lactensis Kuhlm.); BIGNONIACEAE (Jacaranda amazonensis Vattino; Jacaranda brasiliana Pers; Jacaranda paraensis Vattimo; Tabebuia barbata S and w); B O M B A C A CE A E (Catostema albuquerquei Paula; Catostema sclerophyllum Ducke; Matisia ochrocalyx Schum.; Pachira insignis. Schum.; Quararibea ochrocalyx Vischer, Rhodognaphalopsis brevipes A. Robyns; Rhodognaphalopsis minor (Sins.) A. Robyns; Scleronema micranthum Ducke; Scleronema praecox Ducke); BORRAGINACEAE (Cordia nodosa Lam.); BURSERACEAE (Protium lewelynii Macbr; Protium neglectum 
Swartz.); CARYOCARACEAE (Caryocar microcarpum

Ducke; Caryocar villosum

Pers.); CECROPIACEAE (Cecropia purpurascens C.C. Berg;

Cecropia sciadophylla Mart.); CELASTRACEAE (Goniodiscus elaeospermum Kuhlm); CHRYSOBALANACEAE (Couepia longipendula Pilger; Hirtella glabrata Pilger; Licania longistyla Fritsch.; Licania oblongifolia Standl.; Licania octandra Kuntze); CLUSIACEAE (Calophyllum angulare A.C. Smith; Calophyllum brasiliense Camb.; Moronobea pulchra Ducke; Tovomita sp.; Vismia duckei Maguire); CONNARAEAE (Connarus marlenei Forero; Connarus $s p$.); DIALYPETALANTACEAE (Dialypetalanthus fuscescens Kuhl.); DILLENIACEAE (Curatella americana L.); DU CKEODENDRACEAE (Duckeodendron cestroides Kuhlm); EBENACEAE (Diospyros praetermissa Sandw); ELAEOCARPACEAE (Sloanea porphyrocarpa Ducke); ERYTHROXYLACEAE (Erythroxylum macrophyllum Cav.); EUPHORBIACEAE (Aparisthmium cordatum Baill; Croton matourensis Aubl.; Glycidendron amazonicum Ducke; Hevea brasiliensis Muell. Arg.; Hevea guianensis Aubl.; Hura crepitans L.; Joannesia heveoides Ducke; Mabea caudata Pasc. et Hoffm.; Micranda rossiana R.E. Schultes; Micrandropsis scleroxylon W. Rodr.; Richeria laurifolia Baill); FLACOURTIACEAE (Carpotroche crispidentata Ducke; Casearia negrensis Eichl.; Laetia cf. suaveolens Benth.); GOUPIACEAE (Goupia glabra Aubl.); HIPPOCRATEACEAE (Peritassia dulcis Miers);
HUMIRIACEAE (Humiria floribunda Mart.); ICACINACEAE (Emmotum glabrum Miers; Emmotum nitens Miers; Emmotum orbiculatum Miers; Poraqueiba guianensis Aubl.; Poraqueiba paraensis Ducke; Poraqueiba sericea Tul.); LAURACEAE (Aniba affinis Mez; Aniba burchellii Kost.; Aniba ferrea Kubitzki; Aniba hostmanniana Mez.; Aniba santalodora Ducke; Aniba terminalis Ducke; Clinostemon maguireana Kurz; Clinostemon mahuba Kuhlm. et Samp.; Dicypellium manausense W. Rodr.; Endlicheria arunciflora Mez.; Endlicheria sericea Nees; Licaria amara (Mez.) Kosterm.; Licaria canella Kost; Licaria dolichanta Kurz.; Licaria guianensis Aubl.; Licaria vernicosa Kost; Mezilaurus duckei Vd. Werfl.; Mezilaurus decurrens Kost; Mezilaurus synandra Kost.; Nectandra amazonicum Nees; Nectandra spumea Kubitzki; Ocotea canaliculata Mez.; Ocotea costulata Mez.; Ocotea cf. fasciculata Mez.; Ocotea grandifolia Mez; Ocotea guianensis Aubl.; Ocotea pallida (Meiss.) Mez); LECYTHIDACEAE (Asteranthus brasiliensis Desf.; Cariniana decandra Ducke; Corythophora alta R. Knuth.; Couratari stellata A.C. Smith; Eschweilera schomburgkii (Berg) Nied.; Gustavia elliptica Mor); LYTRACEAE (Physocalymma scaberrimum Pohl); MALPIGHIACEAE (Byrsonima chrysophylla H.B.K.; Byrsonima coriaceae Kunth.; Byrsonima fluminensis Ndz.; Byrsonima schomburgkiana Benth.; Pterandra arborea Ducke); 
MELASTOMATACEAE (Bellucia grossularioides Triana; Miconia affinis DC; Miconia poeppigii Triana); MELIACEAE (Carapa guianensis Aubl.; Cedrela odorata L.; Guarea carinata Ducke; Trichilia compacta A.C. Smith.); MONIMIACEAE (Bracteanthus sp.; Siparuna cuspidata A.D.C.); MORACEAE (Brosimum parinarioides Ducke; Brosimum potabile Ducke; Clarisia racemosa R. et P.; Ficus frondosa Standl.; Ficus matsii mathewsii Mig.; Ficus paraensis Mig.; Helicostylis tomentosa Rusby; Naucleopsis caloneura Ducke; Pseudolmedia laevigarpa Trecul.); MYRISTICACEAE (Iryanthera grandis Ducke; Iryanthera macrophylla Warb; Iryanthera tricomis Ducke; Osteophloeum platyspermum Warb; Virola calophylla Spr. et Warb; Virola cuspidata Warb; Virola elongata Warb; Virola guggenheimii W. Rodr; Virola loretensis A.C. Smith; Virola pavonis A.C. Smith; Virola venosa Warb); MYRTACEAE (Eugenia cf. euricheila Berg.; Myrcia magna Legrand Nom); OCHNACEAE (Ouratea discophora Ducke; Ouratea spruceana Engler); OLACACEAE (Curupira tefeensis Black; Minquartia guianensis Aubl.); OPILIACEAE (Agonandra brasiliensis Miers; Agonandra silvatica Ducke); PODOCARPAEAE (Podocarpus sp.); POLYGONACEAE (Coccoloba barbeyana Lindau; Ruprechtia tenuiflora Benth,; Symmeria paniculata Benth.; Triplaris surinamensisv Cham.); RHIZOPHORACEAE (Anisophyllea manausensis Pires et Rodr; Polygonanthus amazonicus Ducke; Sterigmapetalum obovatum Kuhlm.); RUBIACEAE (Bothriospora corymbosa Benth.; Coussarea ampla M. Arg.; Duroia eriopila
L.; Duroia macrophylla Hub.; Isertia hipoleuca Benth.; Isertia rosea Spruce; Pagamea guianensis Aubl.; Palicourea guianensis Aubl.; Remigia cf. ulei Krause); RUTACEAE (Erytrochiton brasiliensis Nees \& Mart.; Euxylophora paraensis Huber; Fagara dellomei Albuq.; Hortia superba Ducke; Myllanthus ulei Cowan; Spathelia excelsa Cowan; Spiranthea guianensis Sandwith; Ticorea longiflora DC; Zanthoxylum compactum Waterm.; Zanthoxylum rhoifolium Lam.); SALICACEAE (Salix martiana Leyb.); SAPOTACEAE (Achrouteria durifructa W. Rodr.; Chrysophyllum oppositum Ducke; Ecclinusa bacuri Aubl. et Pellegr.; Ecclinusa balata Ducke; Ecclinusa ucuquirana branca Aubl.; Elaeoluma glabrescens Mart. et Eichl.; Elaeoluma williamii Aubl. et Pellegr; Glycoxylon inophyllum Ducke; Gymmoluma glabrescens (Mart. et Eiehl.) Aubl.; Manilkara amazonica Standl; Micropholis venulosa Pierre; Micropholis williamii Aubl. et Pellegr; Neolabatia cupraea W. Rodr:; Neoxythece elegans Aubl.; Pouteria gutta Baehni; Pouteria macrophylla (Eym.) Penn.; Pradosia praealta Ducke; Prieurella prieurii Aubr; Ragala ucuquirana branca W. Rodr; Ragala ulei (Krause) Aubr:; Richardella macrophylla Eyma; Richardella manaosensis Aubr, et Pellegr:; Syzygiopsis tarumanensis Pires); SIMARUBACEAE (Simaba guianensis Aubl; Simaruba amara Aubl.); STERCULIACEAE (Sterculia pilosa Ducke; Sterculia pruriens Schum.); TILIACEAE (Apeiba aspera Aubl; Apeiba burchellii Sprague; Apeiba echinata Gaertn; Apeiba membranaceae Spruce; Mollia lepidota Spruce); TRIGONIACEAE 
(Trigonia nivea var. nivea Camb.); VERBENACEAE (Aegiphila intermedia Moldenke; Vitex spongiocarpa Ducke; Vitex triflora Vahl.); VIOLACEAE (Amphirox surinamensis Eichl; Leonia glycicarpa $\mathrm{R}$. et P.; Leonia racemosa Mart.; Paypayrola grandiflora Tul.; Rinorea macrocarpa Kuntze); VOCHYSIACEAE (Erisma sp.; Qualea albiflora Warm.; Qualea cassiquiarensis Spruce ex Warb.; Vochysia guianensis Aubl; Vochysia maxima Ducke; Vochysia inundata Ducke; Vochysia obscura Warm.; Vochysia vismiaefolia Warm.).

Para a preparação de lâminas histológicas foram utilizados cortes de 1620 micrômetros de espessura das faces radial, tangencial e transversal dos corpos de provas. Todos os cortes foram obtidos em microtomos de marca R. Jung. Ag. Mod. 15015 e American Optical Mod. 860. Os cortes foram desidratados em série alcoólica sendo que uns foram mantidos na cor natural, outros coloridos com safranina hidroalcoólica, e demais com hematoxilina e verde-iodo, e por fim montados em resina sintética, entre lâminas e lamínulas. Para as observações microscópicas necessárias, foi utilizado microscópico da marca Carl Zeiss D-7082, com ocular Kpl W 10x/18 e objetivas $2,5 / 0,08,6,3 / 0,16,16 / 0,35$ e 40/0,65. A classificação das inclusões minerais foram baseadas nas normas de IAWA (1989),

\section{RESULTADO E DISCUSSÃO}

Cristais ocorrem no tecido parenquimático axial e radial e nas fibras das espécies examinadas, enquanto que grãos de sílica ocorrem, na grande maioria das vezes, nos raios, poucas vezes no parênquima axial e raríssimas vezes nos elementos fibrosos (Tab.1). No presente estudo foram encontrados grãos de sílica somente nas fibras de Protium neglectum (Burseraceae), enquanto que nas demais espécies ocorrem frequentemente nos raios. Algumas espécies apresentam simultâneamente cristais e sílica no tecido xilemático como, por exemplo, em Pachira insignis (Bombacaceae), onde células parênquimáticas e raios apresentam a ocorrência de tais incrustações. Ressaltase que em nenhuma espécie foi verificada a presença simultânea de sílica e cristal na mesma célula, conforme observou GOTTWALD (1980) em Cordia glabrata (Borraginaceae), onde grãos de silica e cristal ocorrem juntos na mesma célula do raio. Na família Sapotaceae a presença de sílica é constante em quase todos os gêneros examinados, com exceção das espécies Manilkara amazonica, Micropholis pruriense e Achrouteria durifructa. Nesta família, a sílica ocorre mais nas células parenquimáticas dos raios e algumas vezes nas células do parênquima axial e nunca nas fibras. DETTIENE \& JACQUET (1983) não encontraram grãos de silica no xilema de Manilkara bidentata, M.huberi, M.paraensis e M.solimoesensis, mas citam a ocorrência de cristais para essas espécies com exceção de Manilkara solimoesensis. WELLE (1976a) também não observou sílica em várias espécies examinadas do referido gênero.

A não observação de cristais em Manilkara amazonica, incluída no presente estudo, não exclui a possibilidade de sua ocorrência, considerando-se a variabilidade existente em todas as espécies (BAMBER \& LANYON (1960); MARIAUX (1980). 
Tabela 1. Distribuição de cristais e sílica no xilema de madeiras amazônicas.

\begin{tabular}{ccccccc}
\hline \multirow{2}{*}{ ESPÉCIE } & \multicolumn{3}{c}{ CRISTAIS } & \multicolumn{3}{c}{ SÍLICA } \\
\cline { 2 - 7 } & PA & PR & F & PA & PR & F \\
\hline
\end{tabular}

ANACARDIACEAE

Astronium lecointei Ducke

ANNONACEAE

Annona sericea Dun.

Ephedranthus amazonicus R.E.Fries

Rollinia insignis R.E. Fries

\section{APOCYNACEAE}

Ambelania quadrangulares Muell. Arg.

Ambelania tenuiflora Muell. Arg.

Aspidosperma album $R$. Benth

Aspidosperma obscurinervium Azambuja

Couma guianensis Aubl.

Couma utilis Muell. Arg.

Hymatanthus sucuuba Woodson

Macoubea sprucei Mgf.

Malouetia duckei Mgf.

Parahancornia amara Mgf. Monachino

Rauwolfia pentaphylla Ducke

Rauwolfia sellowii Muell. Arg.

Zschokkea lactensis Kuhlm

\section{BOMBACACEAE}

Catostema albuquerquei Paula

Catostema sclerophyllum Ducke

Matisia ochrocalyx Schum.

Pachira insignis K. Schum

Quararibea ochrocalyx Vischer

Rhodognaphalopsis brevipes A. Robyns

Rhodognaphalopsis minor (Sims). A. Robyns

Scleronemia micranthum Ducke

\section{BURSERACEAE}

Protium lewelynii Macbr.

Protium neglectum Swartz

\section{CARYOCARACEAE}

Caryocar microcarpum Ducke

Caryocar villosum Pers.

\section{CHRYSOBALANACEAE}

Couepia longipendula Pilger.

Hirtella glabrata Pilger.

Licania longistyla Fritsch

Licania oblongifolia Standl.

Licania octandra Kuntze

\section{CLUSIACEAE}

Calophyllum brasiliense Camb.

Moronobea pulchra Ducke

Tovomita sp. 
Cont. Tabela 1

\begin{tabular}{ccccccc}
\hline \multirow{2}{*}{ ESPÉCIE } & \multicolumn{3}{c}{ CRISTAIS } & \multicolumn{3}{c}{ SÍLICA } \\
\cline { 2 - 6 } & PA & PR & F & PA & PR & $F$ \\
\hline
\end{tabular}

\section{CONNARACEAE}

Cannarus marlenei Forero

Connarus sp.

\section{DUCKEODENDRACEAE}

Duckeodendron cestroides Kuhlm

\section{EBENACEAE}

Diospyros praetermissa Sandw

\section{ELAEOCARPACEAE}

Sloanea porphyrocarpa Ducke

\section{ERYTHOXYLACEAE}

Erythroxylum macrophyllum Cav.

\section{EUPHORBIACEAE}

Glycidendron amazonicum Ducke Hevea brasiliensis Muell. Arg. Hevea guianensis Aubl. Hura crepitans L. Joannesia heveoides Ducke Mabea caudata Pax. et Hoffm Micrandra rossiana R.E. Schultes Micrandropsis scleroxylon W. Rodr. Richeria laurifolia Baill

\section{FLACOURTIACEAE}

Carpotroche crispidentata Ducke Casearia negrensis Eichl. Laetia cf. suaveolens Benth

\section{HUMIRIACEAE}

Humiria floribunda Mart.

\section{ICACINACEAE}

Emmotum glabrum Miers

Emmotum nitens Miers

Emmotum orbiculatum Miers

Poraqueiba guianensis Aubl.

Poraqueiba paraensis Ducke

\section{LAURACEAE}

Clinostemon maguireana Kurz

Clinostemon mahuba Kuhlm et Samp.

Licaria guianensis Aubl Mezilaurus duckei Vd. Werll Mezilaurus synandra Kost Ocotea canaliculata $\mathrm{Mez}$ Ocotea cf. fasciculata Mez 


\section{\begin{tabular}{llll}
\multirow{2}{*}{ ESPÉCIE } & \multicolumn{2}{c}{ CRISTAIS } & \multicolumn{2}{c}{ SILICA } \\
\cline { 2 - 5 } & PA PR & F PA PR F \\
\hline
\end{tabular}}

\section{LECYTHIDACEAE}

Asteranthus brasiliensis Desf

Cariniana decandra Ducke

Corythophora alta R. Knuth

Eschweilera schomburgkii (Berg) Nied.

\section{MALPIGHIACEAE}

Byrsonima chrysophylla H.B.K.

Byrsonima coriacea Kunth

Byrsonima schomburgkiana Benth

\section{MELIACEAE}

Cedrela odorata $L$.

Trichilia compacta A.C. Smith

\section{MORACEAE}

Clarisia racemosa $R$. et $P$.

Ficus paraensis Miq.

Helicostylis tomentosa Rusby

Naucleopsis caloneura Ducke

Pseudolmedia laevigata Trecul.

\section{MYRTACEAE}

Eugenia cf. lauricheila berg

\section{OLACACEAE}

Curupira tefeensis black

Minquartia guianensis Aubl.

\section{POLYGONACEAE}

Coccoloba barbeyana Liindan.

Ruprechtia tenuiflora Benth

Symmeria paniculata Benth

Triplaris surinamensis Cham.

\section{RHIZOPHORACEAE}

Sterigmapetalum obovatum Kuhlm

\section{RUTACEAE}

Spiranthea guianensis Sandwith

Zanthoxylum compactum Waterm

\section{SALICACEAE}

Salix martiana leyb. 


\begin{tabular}{|c|c|c|c|c|c|c|}
\hline \multirow{2}{*}{ ESPÉCIE } & \multicolumn{3}{|c|}{ CRISTAIS } & \multicolumn{3}{|c|}{ SÍLICA } \\
\hline & PA & PR & $F$ & $\mathrm{PA}$ & PR & $F$ \\
\hline \multicolumn{7}{|l|}{$\overline{\text { SAPOTACEAE }}$} \\
\hline Chrysophllum oppositum Ducke & - & - & - & * & * & * \\
\hline Ecclinusa bacuri Aubl. et Pellegr. & - & - & - & * & * & - \\
\hline Ecclinusa balata Ducke & - & - & - & * & * & - \\
\hline Ecclinusa ucuquirana branca Aubl. & - & - & - & - & * & - \\
\hline Elaeoluma glabrescensMart. et Eichl & - & - & - & - & * & . \\
\hline Elaeoluma williamii Aubl. et Pellegr. & - & - & - & - & * & - \\
\hline Glycoxylon inophyllum Ducke & - & - & - & - & * & - \\
\hline Gymmoluma glabrescens (M. et E.) & - & - & - & - & * & - \\
\hline Micropholis williamii Aubl. et Pellegr. & - & - & - & - & * & - \\
\hline Neolabatia cupraea W. Rodr. & - & - & - & * & * & - \\
\hline Neoxythece elegans Aubl. & - & - & - & - & * & - \\
\hline Pouteria gutta Baehni. & - & - & - & - & * & - \\
\hline Pouteria macrophylla (Eyma) Penn & - & - & - & - & * & - \\
\hline Pradosia praealta Ducke & - & - & - & - & * & - \\
\hline Prieurella prieurii Aubl. & - & - & - & - & * & - \\
\hline Ragala ucuquirana branca W. Rodr. & - & - & - & - & * & - \\
\hline Ragala ulei ( Krause) Aubr. & - & - & - & - & * & - \\
\hline Richardella macrophylla (Krause) Aubr. & - & - & - & - & * & - \\
\hline Richardella manaosensis Eyma & - & - & - & - & * & - \\
\hline $\begin{array}{l}\text { Symmoluma glabrescens A. et Pellegr. } \\
\text { Syzygiopsis tarumanensis Pires. }\end{array}$ & - & - & $\cdot$ & - & * & - \\
\hline \multicolumn{7}{|l|}{ SIMARUBACEAE } \\
\hline Simaba guianensis Aubl. & * & - & - & - & * & - \\
\hline Simaruba amara Aubl. & * & - & - & - & - & - \\
\hline \multicolumn{7}{|l|}{ TILIACEAE } \\
\hline Apeiba aspera Aubl. & - & * & - & - & - & . \\
\hline Apaiba burchellii Sprague & * & * & - & - & - & - \\
\hline Apeiba echinata gaertn & * & * & - & - & - & - \\
\hline Mollia lepidota Spruce & - & * & - & - & - & - \\
\hline \multicolumn{7}{|l|}{ VERBENACEAE } \\
\hline Vitex triflora Vahl. & - & - & - & - & * & - \\
\hline \multicolumn{7}{|l|}{ VIOLACEAE } \\
\hline Leonia racemosa Mart. & - & * & * & - & - & - \\
\hline Paypayrola grandiflora Tul. & - & * & - & - & - & - \\
\hline Rinorea macrocarpa Kuntze & - & * & - & - & - & - \\
\hline \multicolumn{7}{|l|}{ VOCHYSIACEAE } \\
\hline Erisma sp. & * & - & * & - & - & - \\
\hline Qualea albiflora Warm. & * & - & - & - & * & - \\
\hline Qualea cassiquiarensis Spruce ex Warb & * & - & - & - & - & - \\
\hline Vochysia maxima Ducke & * & - & - & - & - & - \\
\hline
\end{tabular}

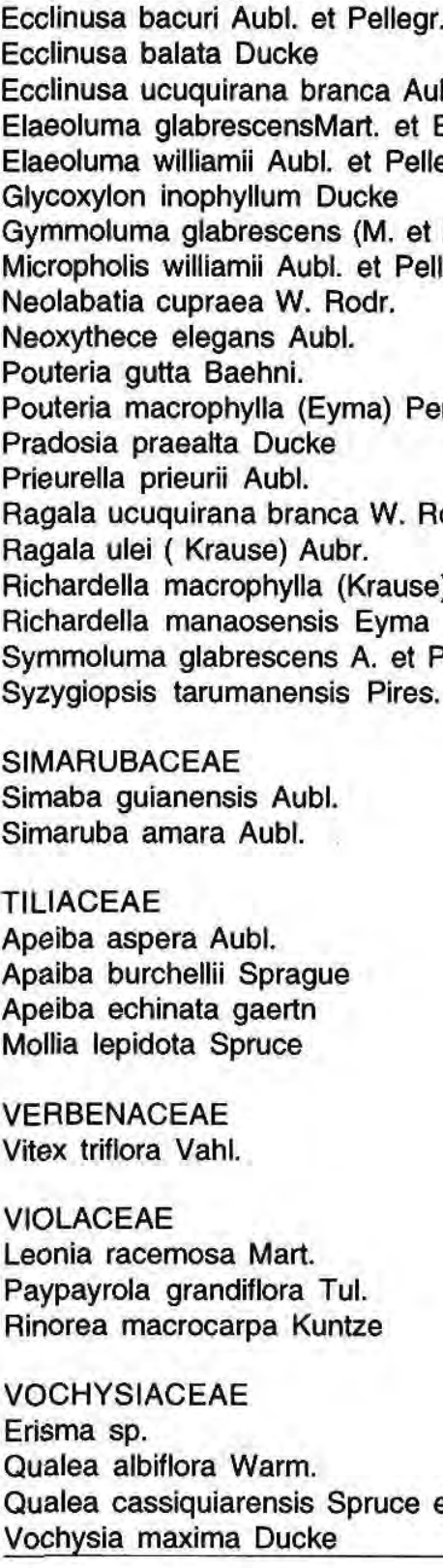

* = Ausência

$\mathrm{PR}=$ Parênquima radial $\quad *$ = Presença

$F=$ Fibras 
$\mathrm{Na}$ única espécie do gênero Micropholis ( $M$. venulosa), incluída no presente estudo, não foi observada a presença de sílica nem de cristal. DETTIENE \& JACQUET (1983), entretanto, citam a ocorrência de silica em oito espécies desse gênero, na maioria das vezes nos raios e, raramente, nas fibras ou parênquima axial. Da mesma maneira aqueles autores citam a ocorrência de silica em Achrouteria pomifera, enquanto que não foram observados grãos de silica e cristal na única espécie de Achrouteria ( $A$. durifructa) examinada. Isto, entretanto, não exclui a possibilidade de sua ocorrência conforme comentado anteriormente. Ao que tudo indica a presença de sílica é uma característica comum a todos os gêneros da família Sapotaceae, com exceção do gênero Manilkara WELLE (1976a); KUKACHKA (1980), (1981a;b;c), DETTIENE \& JACQUET (1983) e pode ser considerada como uma importante característica taxonômica dessa família. WELLE (1976b) cita a ocorrência de silica especialmente nas células do parênquima radial como uma característica taxonômica para a família Chrysobalanaceae, ressaltando que a ocorrência de sílica no parênquima axial dessa familia tem pouco valor taxonômico.

Na família Myristicaceae não foram observados grãos de sílica e cristais, nas espécies dos gêneros Iryanthera (3 espécies), Osteophloeum (1 espécie) e Virola (7 espécies). LISBOA (1989) cita a ocorrência de diminutos cristais em apenas uma amostra de Iryanthera grandis, ao passo que não foram observados cristais nas amostras dessa espécie incluída na presente investigação. A ausência de cristais ou sílica no gênero Virola também foi citada por LOUREIRO et al. (1989) quando do estudo de 24 espécies desse gênero que ocorrem na Amazônia.

A presença de sílica no gênero Vochysia (Vochysiaceae) foi observada por QUIRK (1980) em poucas das 97 espécies desse gênero. No presente estudo não foi verificada a ocorrência de sílica nas espécies examinadas do referido gênero, mas sim a presença de cristais em apenas Vochysia maxima, ao passo que o referido autor não observou cristais nesse gênero.

RICHTER (1982) cita a ocorrência de cristais no gênero Couropita e Couratari (Lecythidaceae), e presença de silica em Couratari. Nesta investigação não foi observada a ocorrência dessa incrustação na única espécie de Couratari (C. stellata) examinada.

Os cristais observados nos raios (parênquima radial) sempre são solitários, podendo estar localizados nas células procumbentes ou eretas. As séries cristalíferas ocorrem sempre nas fibras ou em células de parênquima axial, algumas vezes em longas cadeias de cristais do tipo prismático. Um único cristal do tipo drusa foi encontrado na amostra de Scleronema micranthum (Bombacaceae); nas outras espécies são do tipo prismático.

A Tabela 2 apresenta as amostras que foram observadas e onde não ocorreu a presença de sílica e cristais.

\section{CONCLUSÕES}

Concluindo, o presente trabalho constata a grande incidência de sílica e cristais em madeiras tropicais onde em algumas famílias, como por exemplo Sapotaceae, a presença de uma dessas incrustações é uma característica comum a quase todos os seus gêneros. Resta a 
Tabela 2. Amostras analisadas onde não ocorreu sílica e cristais

ANNONACEAE
Bocageopis multiflora R.E. Fries
Duguetia quiatarensis Benth
Duguetia ulei (Diels) R.E. Fries
Guatteria scytophylla Diels
Pseudoxandra polypheba R.E. Fries
Unonopsis floribunda Diels
Unonopsis guatterioides R.E. Fries
Xylopia aromatica (Lam.) Mart.
Xylopia benthamii R.E. Fries
Xylopia cf. callophylla R.E. Fries

\section{ANTONIACEAE}

Antonia ovata Phol.

\section{APOCYNACEAE}

Aspidosperma oblongum A.DC.

Himatanthus attenuata Woodson

Lacmellea cf. lactescens Kuhlm

Macoubea guianensis Aubl.

Neocouma ternstroeniaceae (Muell. Arg.) Pierre

Rauwolfia duckei Muell. Arg.

\section{BIGNONIACEAE}

Jacaranda amzonensis Vattimo

Jacaranda brasiliana Pers.

Jacaranda paraensis Vattimo

Tabebuia barbata Sandw

\section{BOMBACACEAE}

Scleronema praecox Ducke

\section{BORRAGINACEAE}

Cordia nodosa Lam.

\section{CECROPIACEAE}

Cecropia purpurascens C.C. Berg

Cecropia sciadophylla Mart.

\section{CELASTRACEAE}

Goniodiscus elaeospermum Kuhim

\section{CLUSIACEAE}

Calophyllum angulare AC. Smith

Vismia duckei Maguire

\section{DIALYPETALANTHACEAE}

Dialupetalanthus fuscescens Kuhlm

\section{DILLENIACEAE}

Cuaratella americana $\mathrm{L}$.

\section{EUPHORBIACEAE}

Aparisthmium cordatum Baill

Croton matourensis Aubl.

GOUPIACEAE

Goupia glabra Aubl.

HIPPOCRATEACEAE

Peritassia dulcis Miers

ICACINACEAE

Poraqueiba sericeae Tul.

\section{LAURACEAE}

Aniba affinis $\mathrm{Mez}$

Aniba burchellii Kost

Aniba ferrea Kubitzki

Aniba hostmanniana Mez

Aniba santalodora Ducke

Aniba terminalis Ducke

Dicypellium manausense W. Rodr.

Endlicheria arunciflora Mez

Endlicheria sericea Nees

Licaria amara (Mez.) Kost.

Llcaria canella Kost

Licaria dolichanta Kunz

Licaria vernicosa Kost

Mezilaurus decurrens Kost

Nectandra amazonicum Nees

Nectandra spumea Kubitzki

Ocotea costulata Mez

Ocotea grandigolia $\mathrm{Mez}$

Ocotea guianensis Aubl.

Ocotea pallida

\section{LECYTHIDACEAE}

Couratari stellata A.C. Smith

Gustavia elliptica Mor

\section{LYTRACEAE}

Physocalymma scaberrimum Pohl

\section{MALPIGHIACEAE}

Byrsonima fluminensis $\mathrm{Ndz}$.

Pterandra arborea Ducke

\section{MELASTOMATACEAE}

Bellucia grossularioides Trina

Miconia affinis DC.

Miconia poeppigii Triana 
Carapa guianensis Aubl.

Guarea carinata Ducke

\section{MONIMIACEAE}

Bracteanthus sp.

Siparuna cuspidata A. DC.

MORACEAE

Brosimum parinarioides Ducke

Brosimum potabile Ducke

Ficus frondosa Standl

Ficus matsii mathewsii Miq.

\section{MYRISTICACEAE}

Iryanthera grandis Ducke

Iryanthera macrophylla Warb

Iryanthera tricornis Ducke

Osteophloeum platyspermum Warb.

Virola calophylla Spr, et Warb.

Virola cuspidada Warb.

Virola elongata Warb.

Virola guggenheimii W. Rodr.

Virola loretensis A.C. Smith

Virola pavonis A.C. Smith

Virola venosa Warb.

\section{MYRTACEAE}

Myrcia magna Legrand Nom

\section{OCHNACEAE}

Ouratea discophora Ducke

Ouratea spruceana Engler

\section{OPILIACEAE}

Agonandra brasiliensis Miers

Agonandra silvatica Ducke

\section{PODOCARPACEAE}

Podocarpus sp.

\section{RHIZOPHORACEAE}

Anisophyllea manausensis Pires et Rodr.

Polygonanthus amazonicus Ducke

\section{RUBIACEAE}

Bothriospora corymbosa Benth

Coussarea ampla M. Arg.
Duroia eriopila L.

Duroia macrophylla Hub

Isertia hipoleuca Benth

Isertia rosea Spruce

Paganea guianensis Aubl

Palicourea guianensis Aubl.

Remigia cf. ulei Krause

\section{RUTACEAE}

Erytrochiton brasiliensis Nees \& Mart.

Euxylophora paraensis Huber

Fagara dellomei Albuq.

Hortia superba Ducke

Myllanthus ulei Cowan

Spathelia excelsa Cowan

Ticorea longiflora DC

Zanthoxylum rhoifolium Lam

\section{SAPOTACEAE}

Achrouteria durifructa W. Rodr. Manilkara amazonica Standl

Micropholis venulosa Pierre

\section{STERCULIACEAE}

Sterculia pilosa Ducke

Sterculia pruriens Schum

\section{TILIACEAE}

Apeiba membranaceae Spruce

TRIGONIACEAE

Trigonia nivea var. nivea Camb

\section{VERBENACEAE}

Aegiphila intermedia Moldenke

Vitex spongiocarpa Ducke

\section{VIOLACEAE}

Amphirox surinamensis Eichl

Leonia glycicarpa $\mathbf{R}$. et $\mathbf{P}$.

\section{VOCHYSIACEAE}

Vochysia guianensis Aubl.

Vochysia inundata Ducke

Vochysia obscura Warm

Vochysia vismiaefolia Warm 
realização de novos estudos sobre a questão para verificar-se o valor dessas inclusões como características taxonômicas para algumas familias, bem como seu nível de influência sobre o processamento mecânico das madeiras portadoras das mesmas.

\section{AGRADECIMENTOS}

Agradecemos a todos que contribuiram para a execução deste trabalho, em especial a direta participação da Acadêmica de Engenharia Florestal GABRIELA BALBI, pela paciente observação das lâminas histológicas em microscópio, e a colega Vania Maria Oliveira da Camara Lima pela digitação do trabalho.

\section{Bibliografia Citada}

BAMBER, R. K.; LANYON, J. W. 1960. Silica deposition in several woods of new South Wales. Tropical woods, 113: 48-53.

DETIENNE, P.; JACQUET, P. 1983. Atlas d'identification des bois de l'amazonie et des regions voisines. Centre Technique Forestier Tropical . France, 640 p.

FREITAS, J. A.; VASCONCELLOS, F. J.; de ; SILVA, A. C. 1992. Deposição de sílica e cristais no xilema de espécies tropicais nas familias FABACEAE e MIMOSACEAE. Anais do I Congresso Internacional de Compensados de Madeiras Tropical Associação Brasileira da Indústria de Madeira Compensada e Industrializada ABIMCI/International Tropical Timber Organization - ITTO, Manaus: 233-238.

GOTTWALD, H. 1980. "Louro Preto" - found to be the first silica bearing Cordia (Cordia glabrata, Bonaginaceae). IAWA Bulletin n.s., 1(1-2): 55-58.

GOURLAY, I. D. ; KANOWSKI, P.J. 1991. Marginal parenchyma bands ans crystalliferous chains as indicatorsof age in African Acacia species. IAWA Bulletin n. s., 12(2): 187-194.

IAWA. 1989. List of microscopic features for hardwood identification. IAWA Bulletin, 10 (3): 219-232.

KOEPPEN, R. C. 1980. Silica bodies in wood of arborescent Leguminosae. IAWA Bulletin $\mathrm{n}$, s., 1(4): 180-184.

KUKACHKA, B.F, 1980. Wood anatomy of the Neotropical Sapotaceae. XIV. Elaeoluma. Research Paper FLP 358. Madison, WIS.

-..- - 1981a. Wood anatomy of the Neotropical Sapotaceae. XXII. Pradosia. Research Paper FLP 373. Madison, WIS.

1981b. Wood anatomy of the Neotropical Sapotaceae. XXIV. Ecclinusa. Research Paper. FLP 395. Madison, WIS. 1981c. Wood anatomy of the Neotropical Sapotaceae. XXV. Ragala, Research Paper FLP 396. Madison, WIS.

LAROCHE, J. 1977. La silice et les plantes superieures. Rev. Cyt. Biol. Veg. 40: 15-45.

LISBOA, P.L.B.1989 . Aspectos da anatomia sistemática do lenho de Iryanthera Warb. (Myristicaceae). Boletim do Museu Paraense Emílio Goeldi. Série Botânica, 5(2): 83-134.

LOUREIRO, A. A.; FREITAS, M. C.; VASCONCELLOS, F.J. 1989, Estudo anatômico de 24 espécies do gênero Virola (Myristicaceae) da Amazônia. Acta Amazonica, 19: 415-465.

MARIAUX, A. 1980. Formation of silica grains in wood as a function of growth rate. IAWA Bulletin n. s. 1(3): 140-142.

QUIRK, J.T. 1980. Wood anatomy of the Vochysiaceae. IAWA Bulletin n. s., 1(4); 172-179.

RECORD, S. J. 1927. Occurrence of calcium carbonate deposits in wood. Tropical Woods., 12: 22-26.

RICHTER, H. G, 1982. The wood structure of Couratari Aubl. and Couropita Aubl. (Lecythidaceae). IAWA Bulletin n.s., 3(1): 45-55.

SCURFIELD, G; ANDERSON, C.A.; SEGNIT, E.R. 1974. Silica in woody stems. Australian Journal of Botany, 22: 211-231. 
SHARMA, M. ; RAO, K. R. 1970. Investigations on the occurrence of silica in Indian timbers. Indian Forester. 96: 740-754.

VASCONCELLOS, F. J. de; SILVA, A. C. ; FREITAS, J.A. 1993. Deposição de silica e cristais no xilema de espécies tropicais da família CAESALPINIACEAE. Revista Árvore, Viçosa, 17 (3): 369-374.
WELLE, B.J.H. 1976a. Silica grains in woody plants of the neotropics especially Surinam. Leiden Botanical Series 3: 107-142.

WELLE, B. J. H. 1976b. On the occurrence of silica grains in the secondary xylem of the Chrysobalanaceae. IAWA Bulletin 2: 19-29. 